\title{
Rapid On-site Evaluation (ROSE) for Fine Needle Aspiration of Thyroid: Is It Helpful?
}

\author{
Ahmed K. Aly ${ }^{1 *}$, Mahmoud A. Ali ${ }^{1}$, Apoorva Sharma ${ }^{1}$, Matthew A. Gubbels ${ }^{1}$, \\ Xing Zhao ${ }^{2}$, Ayahallah Ahmed ${ }^{1}$, Sarah Aurit ${ }^{3}$, Joseph Stavas ${ }^{1}$ \\ ${ }^{1}$ Radiology Department, School of Medicine, Creighton University, Omaha, Nebraska,United States. \\ ${ }^{2}$ Pathology Department, School of Medicine, Creighton University, Omaha, Nebraska,United States.
}

${ }^{3}$ Statistician at Surgery Department, School of Medicine, Creighton University, Omaha, Nebraska, United States

Received 24 October 2020; Revised 28 January 2021; Accepted 12 February 2021; Published 01 March 2021

\begin{abstract}
Introduction: Thyroid nodules are very common. Many are detected incidentally due to increased head and neck imaging. The majority are benign; however, malignancy can't be excluded in many cases and tissue sampling is needed. Ultrasound guided fine needle aspiration is an easy and effective way to sample g thyroid nodules. Inadequate sampling was reported in $10-40 \%$ of the cases. Rapid On-site Evaluation (ROSE) was proposed to assess obtained sample for adequacy. The aim of this study is to identify the benefit of applying ROSE with US-FNA of thyroid nodules within our institution. Materials and methods: Patients who underwent FNA for thyroid nodules with ROSE availability documented in their procedure note between January 2017 to December 2018 were retrospectively included. All procedures were done by experienced radiologists. Aspirated material was Diff Quik stained for immediate evaluation. The final cytological diagnosis and specimen adequacy was based on The Bethesda system for reporting thyroid cytopathology. Specimen adequacy was compared between ROSE and non-ROSE groups. Results: 442 thyroid nodules were biopsied. ROSE was available for 65 nodules. Non-diagnostic rate with ROSE was $10.8 \%$ compared to $13.8 \%$ without ROSE with the difference being statistically insignificant. ROSE availability improved sample adequacy of nodules less than $3 \mathrm{~cm}$ with statistically significant difference of $100.0 \%$ with ROSE vs. $87 \%$ without ROSE. Conclusion: The current study does not justify the routine use of ROSE. However, ROSE availability is beneficial with smaller sized thyroid nodules and less experienced radiologists performing the procedure.
\end{abstract}

Keywords: ROSE; FNA; Ultrasound; Cytopathology; Bethesda System.

\section{Introduction}

Thyroid nodules are one of the most common clinical findings, their prevalence depends on the population studied and the tool used for screening [1]. As the use of neck imaging increases, the number of incidentally discovered increases [2]. Approximately 62,450 new thyroid cancer cases were diagnosed in the United States during 2015 with this being the $5^{\text {th }}$ leading cause of cancer in women [3]. Around 3000 ultrasounds exams done annually in our institution with a lot of thyroid nodules demonstrated. Most of those nodules would turn out to be benign, however malignancy needs to be excluded in many cases [4].

* Corresponding author: ahmedaly@creighton.edu

\section{doi http://dx.doi.org/10.28991/SciMedJ-2021-0301-1}

$>$ This is an open access article under the CC-BY license (https://creativecommons.org/licenses/by/4.0/).

(C) Authors retain all copyrights. 
When tissue sampling is required, ultrasound guided fine needle aspiration [US-FNA] represents an easy and effective way of getting the needed tissue with minimal risk [5]. Despite the use of ultrasound to guide our choice for biopsy site, obtaining inadequate sample for diagnosis has been one of the most frustrating consequences of this procedure, mainly due to obtaining few or no cells which is reported to occur in $10-40 \%$ of cases $[6,7]$. This can result in many cases in repeating the procedure which subsequently contributes towards increasing cost and patient anxiety; it can also lead to treatment delay [8].

Rapid On-Site Evaluation (ROSE) of the obtained sample was proposed to determine the presence of adequate cells in the obtained specimen. Multiple studies around the world has demonstrated beneficial effect of this strategy in decreasing the rate of inadequate specimen with US-FNA at multiple sites, including thyroid [7, 9-12]. Some even suggested using tele-cytopathology to overcome the shortage of onsite cytopathologist at some sites [13]. However, this comes with the price of prolonging the procedure time and increasing the cost, with some studies suggesting no overall benefit $[14,15]$.

In this study we retrospectively analyzed the adequacy rate of thyroid FNA samples with and without ROSE implementation. Adequacy of the collected sample was determined according to the Bethesda scoring system. We also analyzed ROSE availability difference with age, sex, and nodule size. Our objective was to identify the benefit of using ROSE with US-FNA of thyroid nodules in our institution.

\section{Methods}

\subsection{Study Population and Design}

All data were collected retrospectively from our electronic medical records after obtaining the approval of the Biomedical Institutional Review Board. All patients who underwent US-FNA for thyroid nodules between January 2017 to December 2018 were included. The pathology and procedure reports were collected for all the patients. If the availability of ROSE was not clear in the procedure reports the patient was excluded (Figure 1).

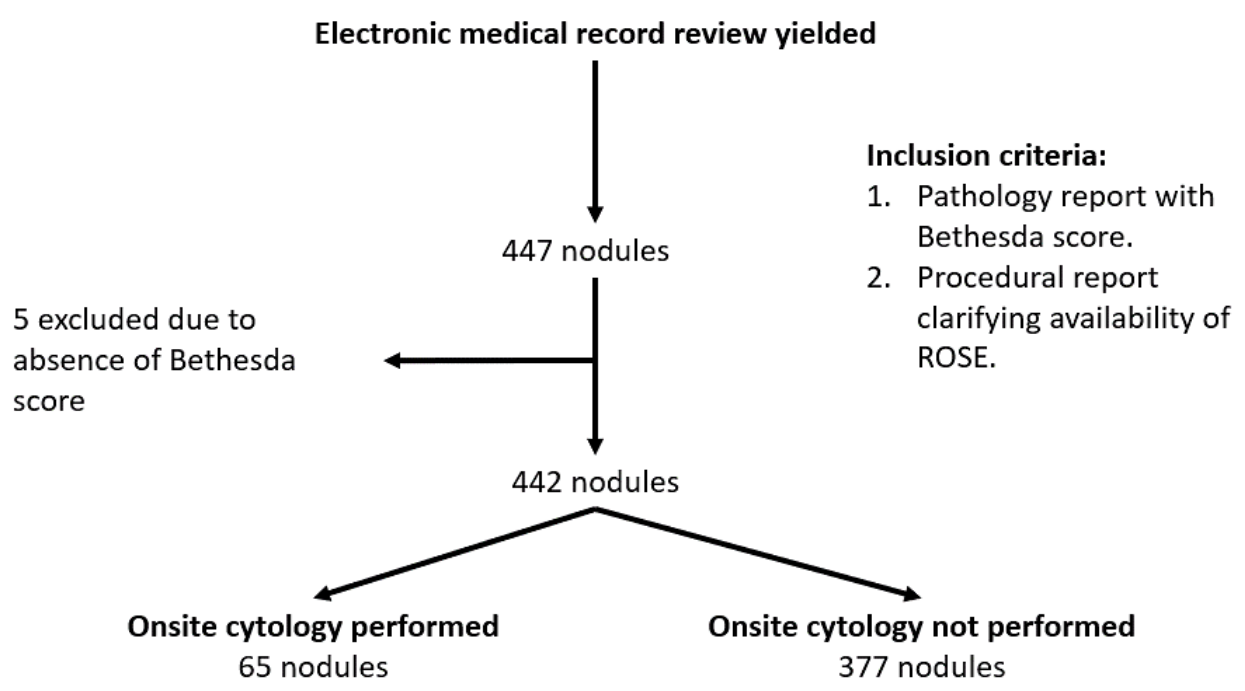

Figure 1. Flowchart highlighting study design and patient selection criteria.

\subsection{Procedure}

The patient was placed in supine position with slight neck hyperextension. Ultrasound examination was performed to identify the best access site. The skin was then cleaned with chlorhexidine and the area was draped in sterile fashion. One percent Lidocaine was used in every case for local anesthesia to anaesthetize the skin and the expected needle track under ultrasound guidance using linear high frequency probe. Under ultrasound guidance a free hand technique was used to advance 22-25 G needle mounted on $10 \mathrm{cc}$ empty syringe towards the target nodules and the sample was aspirated. This was repeated 5 more times or less in case sample deemed adequate by the on-site pathologist whenever available.

Aspirated material was then placed on to a glass slide and smeared. In cases where ROSE was available, two smear slides of each pass were prepared. One slide was air-dried and stained by Diff Quik and examined under light microscopy to assess for adequacy. The second slide was then fixed with $95 \%$ ethyl alcohol and submitted to the pathology lab for PAP stain. The aspirate from last 1-2 passes was placed in Afirma test tube and saved for future genetic study if needed. All the procedures were performed by radiologists with more than 10 years of experience in ultrasound guided procedures. 


\subsection{Outcomes}

The availability of ROSE in each case was documented based on the procedure report. The final cytological diagnosis was based on The Bethesda system for reporting thyroid cytopathology [16] and was classified into one of 6 categories: (I) nondiagnostic or unsatisfactory; (II) benign; (III) atypia of undetermined significance or follicular lesion of undetermined significance; (IV) follicular neoplasm or suspicious for a follicular neoplasm; (V) suspicious for malignancy; and (VI) malignant. Samples were considered inadequate if they were classified as I and were considered adequate otherwise.

\subsection{Statistical Methods}

Discrete variables are presented as frequency and proportion and continuous variables are presented as median and Interquartile Range (IQR). Patient and nodule data were stratified by availability of ROSE and by the binary designation of adequacy. The expected cell frequencies of discrete variables were examined in order to select the chisquare or Fisher's exact test; regardless of distribution of continuous variables, comparisons were with the MannWhitney test.

Multiple imputation was utilized to impute missing age and maximum nodule size by fully conditional specification methods [17]. A mixed effects multivariable binary logistic regression model with robust sandwich estimator to control for inflation of Type I error was selected to examine predictors of adequacy while accommodating the clustering of multiple nodules within patients [18]. Restricted cubic splines with three knots at equally placed percentiles were constructed to model age and maximum nodule size. SAS version 9.4 was used for all analyses; $\mathrm{P}<0.05$ indicated statistical significance [19].

\section{Results}

We obtained data from 447 nodules and omitted five due to missing Bethesda score and adequacy status for a total of 442 nodules associated with 371 patients. Demographic and clinical characteristics from patients and nodules stratified by the availability of ROSE are presented in Table 1 . The majority of patients were female (79.8\%) and had a median age of 59 years (IQR: 46 years to 68 years). Of the 442 nodules, maximum size was missing for a total of 90 nodules. The median nodule size was $2.4 \mathrm{~cm}$ and were predominantly classified as benign (Bethesda score $=$ II; (73.8\%). ROSE was available for 62 patients (16.7\%) and 65 nodules (14.7\%). A total of 59 biopsies (13.3\%) were classified as Bethesda I (nondiagnostic or unsatisfactory), and there was no statistical evidence of a difference associated with ROSE availability. In general, there was no ROSE availability difference associated with patient age and biological sex, maximum nodule size, and overall adequacy. It was found that $383(86.7 \%)$ of nodules were adequate from 320 patients $(86.3 \%)$. There was no evidence of an adequacy difference in relation to patient age and biological sex and maximum nodule size.

Table 1. Baseline characteristics of patients stratified by presence of rapid onsite evaluation

\begin{tabular}{|c|c|c|c|c|}
\hline \multirow{2}{*}{ Variable of Interest } & & No ROSE & ROSE & \multirow{2}{*}{$\boldsymbol{P}$} \\
\hline & & {$[n=309]$} & {$[n=62]$} & \\
\hline Age, years, median (IQR) & $n=371$ & $59[45-68]$ & $61[52-74]$ & 0.122 \\
\hline Biological Sex, $n(\%)$ & $n=371$ & & & 0.380 \\
\hline Female & & $244[79.0]$ & $52[83.9]$ & \\
\hline Male & & $65[21.0]$ & $10[16.1]$ & \\
\hline Maximum Nodule Size, cm, median (IQR) & $n^{*}=352$ & $2.4[1.7-3.4]$ & $2.4[1.6-3.1]$ & 0.574 \\
\hline Bethesda Score, $n(\%)$ & $n *=442$ & & & 0.254 \\
\hline I & & $52[13.8]$ & $7[10.8]$ & \\
\hline II & & 278 [73.7] & 48 [73.9] & \\
\hline III & & $29[7.7]$ & $4[6.1]$ & \\
\hline IV & & $6[1.6]$ & $0[0.0]$ & \\
\hline V & & $5[1.3]$ & $2[3.1]$ & \\
\hline VI & & $7[1.9]$ & $4[6.1]$ & \\
\hline Adequacy, $n(\%)$ & $n^{*}=442$ & & & 0.508 \\
\hline No & & $52[13.8]$ & $7[10.8]$ & \\
\hline Yes & & $325[86.2]$ & $58[89.2]$ & \\
\hline
\end{tabular}

*By Individual Nodule 
We also stratified clinical and demographic characteristics by adequacy of cell collection at the patient and nodule level (Table 2). In comparison of adequate collection to inadequate collection, there was no difference in median age (59 years vs. 57 years; $P=0.627)$ or female biological sex $(79.4 \%$ vs. $82.4 \% ; P=0.623)$. Similarly, it was also found that there was no difference in maximum nodule size $(2.4 \mathrm{~cm}$ vs. $2.2 \mathrm{~cm} ; P=0.356)$.

Table 2. Multivariable Mixed Effects Binary Logistic Regression for Prediction of Adequacy

\begin{tabular}{ccccc}
\hline Variable of Interest & Odds Ratio & LL 95\% CI & UL 95\% CI & $\boldsymbol{P}$ \\
\hline $\begin{array}{c}\text { Biological Sex } \\
\text { Male }\end{array}$ & \multicolumn{4}{c}{ Reference } \\
Female & 0.70 & 0.30 & 1.63 & 0.411 \\
ROSE & \multicolumn{2}{c}{ Reference } \\
No & \multicolumn{2}{c}{1.79} & 0.591 \\
Yes & 0.80 & 0.36 & 1.79 \\
\hline
\end{tabular}

Results from the mixed effects binary logistic regression model are presented in Table 3 . It was found after adjusting for age, maximum nodule size, and biological sex, there was no statistical evidence of a relationship with ROSE availability and adequacy $(\mathrm{OR}=0.80,95 \% \mathrm{CI}: 0.36$ to $1.79 ; P=0.591)$.

Table 3. Multivariable Mixed Effects Binary Logistic Regression for Prediction of Adequacy

\begin{tabular}{ccccc}
\hline Variable of Interest & Odds Ratio & LL 95\% CI & UL 95\% CI & $\boldsymbol{P}$ \\
\hline Biological Sex & \multicolumn{5}{c}{ Reference } \\
Male & 0.70 & 0.30 & 1.63 & 0.411 \\
Female & \multicolumn{5}{c}{ Reference } \\
ROSE & \multicolumn{5}{c}{1.79} \\
No & 0.80 & 0.36 & 0.591 \\
Yes &
\end{tabular}

Rate of adequacy was also compared among smaller size nodules. When examining nodules with maximum diameter of $2 \mathrm{~cm}$, it was found that ROSE availability in comparison to none was associated with increased adequacy $(100.0 \%$ vs. $83.3 \% ; P=0.042)$. In a similar comparison for nodules with a maximum diameter of $3 \mathrm{~cm}$, there was also evidence of an increased adequacy rate (100.0\% vs. $87.0 \% ; \mathrm{P}=0.019)$ (Figure 2$)$.

Onsite cytology performed

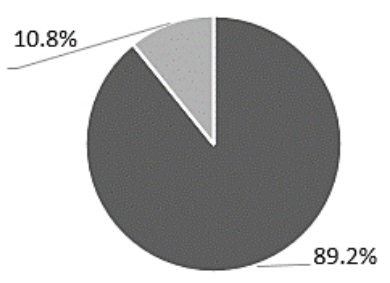

घ Adequate inadequate

Onsite cytology not performed

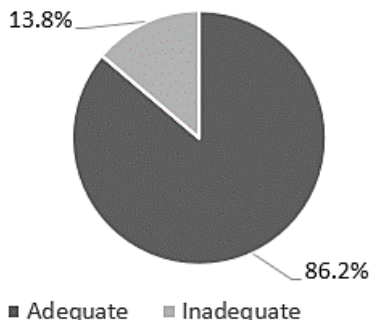

All nodules
Onsite cytology performed

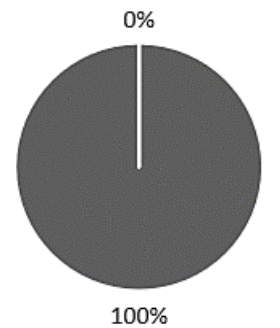

- Adequate $\square$ Inadequate

Onsite cytology not performed

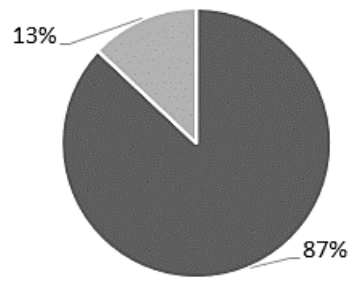

घ Adequate Inadequate
Onsite cytology performed

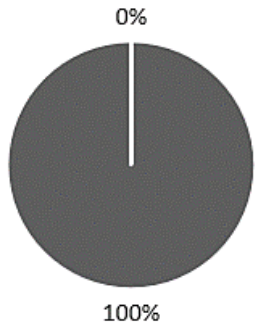

a Adequate $\square$ Inadequate

Onsite cytology not performed

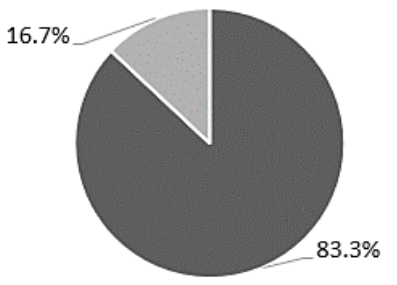

n Adequate Inadequate

Nodules $\leq \mathbf{3}$

Figure 2. The effect on performing onsite cytological exam on obtaining adequate sample in different nodule sizes 


\section{Discussion}

US-FNA is the most commonly available way to evaluate thyroid nodules, however obtaining inadequate samples remain one of the challenging aspects of this procedure with rates up to $40 \%$ reported in the literature [7] Ideally, non-diagnostic samples should not exceed 10\% [8]. ROSE was suggested to improve the adequacy rate of obtained samples. The reported literature appears to be controversial about that. Some studies reported significant improvements after application of ROSE with up to 30\% drop in the inadequacy rate [7, 10, 20]. Others failed to find a statistically significant improvement in the adequacy rates after application of ROSE [8, 14]. This was attributed to different practice patterns, radiologist's experience and other factors. There is debate in literature about the cost effectiveness of ROSE. Barriers to ROSE includes additional costs charged by cytology to fees of the procedure. At the same time ROSE requires cytologist or pathologist to be available at FNA site which is not always available. Also, ROSE can increase the overall procedure time. Despite that some might argue that ROSE benefits may outweigh costs by decreasing the number of FNA repeats [21]. Other institutes implemented tele cytopathology for suspicious nodules to overcome shortage of onsite cytopathologist with relatively good results, however with increased cost and procedure time $[13,22]$.

In their meta-analysis, Witt et al. concluded that ROSE was associated with improved sample adequacy rate from 83 to $92 \%$ with the largest effect noted in studies with lowest initial -without ROSE- adequacy rates [11]. A large study conducted by Pastorello et al. reported significant improvement in adequacy rates from $69.4 \%$ without ROSE to 93.4\% with ROSE and a decrease in the mean number of thyroid needle passes from 3.0 to 1.5 [20]. Another study by Chamorro et al. (2018) evaluated 552 thyroid nodules with $30.5 \%$ decrease in non-diagnostic samples after implementation of ROSE [7].

Other studies reported more benefit in cases of less experienced radiologist and small size nodules [8, 23]. A Chinese study conducted on 1103 thyroid nodules did not show a statistically significant difference in sample adequacy between the two subgroups. However, ROSE was found to increase sample adequacy in subset of thyroid nodules including sub centimetric, mixed solid and cystic nodules and nodules with macrocalcification. The mean number of thyroid passes was reduced from 2.8 without ROSE to 1.7 with ROSE [8]. Another study by Ghofrani et al. was performed on a total of 1502 thyroid nodules; 981 were performed under ultrasound guidance and 521 by palpation. The non-diagnostic rate was 5.3\% with ROSE compared to $10.5 \%$ without ROSE in the entire group. However, there was no statistically significant difference in non-diagnostic sample rates among US guided subgroup. Interestingly, among less experienced radiologists the non-diagnostic rates were reduced from 13 to $4.5 \%$, suggesting that the value of ROSE might vary according to the experience of radiologist performing the procedure [23].

In our study the non-diagnostic rate with ROSE was $10.8 \%$ compared to $13.8 \%$ without ROSE a statistically insignificant result. This can be due to the low rate of non-diagnostic samples. This can be attributed to the low rate of non-diagnostic samples at study initiation. All radiologists who performed our cases had more than 10 years' experience in ultrasound guided procedures and that might be another factor. We then investigated the effect of ROSE in certain subsets based on the nodule maximum diameter, ROSE was more helpful in smaller nodules which is concordant with previously reported results in the literature.

Due to the retrospective nature of our study we were not able to accurately and reliably measure the effect of ROSE on number of needle passes and overall time of the procedure. However, it was subjectively reported in multiple cases that the procedure was stopped before completing the standard 6 passes when adequate samples were obtained. Another factor that we didn't evaluate is the cost of doing ROSE in all cases compared to the cost of repeat biopsy in cases were inadequate sample was obtained.

\section{Conclusion}

In summary, the beneficial effect of ROSE is controversial, and this might not justify its routine application in all cases. ROSE was found to be the most helpful with small sized nodules or nodules that are more difficult to sample for less experienced radiologists. Other studies suggested that the value of ROSE in decreasing FNA repeats outweighs the additional costs of ROSE related fees. Additional reported values in literature are decreasing the number of needle passes needed to obtain adequate sample and thus degree of patient discomfort. The decision to implement ROSE should be tailored according to the availability of resources and the patient population. It should be a case by case decision based on nodule characteristics and the radiologist's experience.

\section{Declarations}

\subsection{Author Contributions}

M.A.A. conceived the idea; A.K.A, M.A.A., X.Z., A.S., M.A.G. and J.S. contributed to the study conception and design; J.S. was involved in planning and supervised the work; A.K.A., M.A.A., X.Z, S.A., A.S. and M.A.G. were involved in data acquisition, organization and drafting; X.Z. provided details about the methods used in processing 
and evaluation of tissue samples. SA. planned and performed the statistical analysis; S.A., A.K.A., M.A.A. and J.S. contributed to data analysis and interpretation; A.A. and A.K.A. completed the literature search and review; A.A., A.K.A., X.Z, S.A., A.S., and J.S. drafted the manuscript and designed the tables. A.A. and J.S. performed the final critical revisions. All authors discussed the results and commented on the manuscript. All authors have read and agreed to the published version of the manuscript.

\subsection{Funding}

The author(s) received no financial support for the research, authorship, and/or publication of this article.

\subsection{Ethical Approval}

This study was reviewed and approved by the biomedical Institutional Review Board (IRB) at the Creighton University.

\subsection{Data Availability Statement}

The data presented in this study are available on request from the corresponding author.

\subsection{Conflict of Interest}

The authors declare that they have no known competing financial interests or personal relationships that could have appeared to influence the work reported in this paper.

\section{References}

[1] Dean, D. S., \& Gharib, H. (2008). Epidemiology of thyroid nodules. Best Practice \& Research Clinical Endocrinology \& Metabolism, 22(6), 901-911. doi:10.1016/j.beem.2008.09.019.

[2] Hoang, J. K., Langer, J. E., Middleton, W. D., Wu, C. C., Hammers, L. W., Cronan, J. J., ... Berland, L. L. (2015). Managing Incidental Thyroid Nodules Detected on Imaging: White Paper of the ACR Incidental Thyroid Findings Committee. Journal of the American College of Radiology, 12(2), 143-150. doi:10.1016/j.jacr.2014.09.038.

[3] National Cancer Institute (2020). American Society of Clinical Oncology. Definitions. Code C39319. doi:10.32388/wgy03s.

[4] Haugen, B. R. (2016). 2015 American Thyroid Association Management Guidelines for Adult Patients with Thyroid Nodules and Differentiated Thyroid Cancer: What is new and what has changed? Cancer, 123(3), 372-381. doi:10.1002/cncr.30360.

[5] Egset, A. V., Holm, C., Larsen, S. R., Nielsen, S. H., Bach, J., Helweg-Larsen, J. P., ... Godballe, C. (2017). Risk of malignancy in fine-needle aspiration biopsy in patients with thyroid nodules. Danish Medical Journal, 64(2), A5320.

[6] Yeon, J. S., Baek, J. H., Lim, H. K., Ha, E. J., Kim, J. K., Song, D. E., Lee, J. H. (2013). Thyroid Nodules with Initially Nondiagnostic Cytologic Results: The Role of Core-Needle Biopsy. Radiology, 268(1), 274-280. doi:10.1148/radiol.13122247.

[7] Medina Chamorro, F. M., Calle, J. A., Stein, J. E., Merchancano, L., Mendoza Briñez, A. M., \& Pulido Wilches, A. A. (2018). Experience of the Implementation of Rapid On-Site Evaluation in Ultrasound-Guided Fine-Needle Aspiration Biopsy of Thyroid Nodules. Current Problems in Diagnostic Radiology, 47(4), 220-224. doi:10.1067/j.cpradiol.2017.06.009.

[8] Jiang, D., Zang, Y., Jiang, D., Zhang, X., \& Zhao, C. (2018). Value of rapid on-site evaluation for ultrasound-guided thyroid fine needle aspiration. Journal of International Medical Research, 47(2), 626-634. doi:10.1177/0300060518807060.

[9] Breeze, J., Poller, D. N., Gibson, D., Tilley, E. A., Cooke, L., Soar, E., \& Repanos, C. (2013). Rapid on-site assessment of specimens by biomedical scientists improves the quality of head and neck fine needle aspiration cytology. Cytopathology, 316321. doi:10.1111/cyt.12106.

[10] De Koster, E. J., Kist, J. W., Vriens, M. R., Borel Rinkes, I. H. M., Valk, G. D., \& de Keizer, B. (2016). Thyroid UltrasoundGuided Fine-Needle Aspiration: The Positive Influence of On-Site Adequacy Assessment and Number of Needle Passes on Diagnostic Cytology Rate. Acta Cytologica, 60(1), 39-45. doi:10.1159/000444917.

[11] Witt, B. L., \& Schmidt, R. L. (2013). Rapid Onsite Evaluation Improves the Adequacy of Fine-Needle Aspiration for Thyroid Lesions: A Systematic Review and Meta-Analysis. Thyroid, 23(4), 428-435. doi:10.1089/thy.2012.0211.

[12] Schmidt, R. L., Witt, B. L., Lopez-Calderon, L. E., \& Layfield, L. J. (2013). The Influence of Rapid Onsite Evaluation on the Adequacy Rate of Fine-Needle Aspiration Cytology. American Journal of Clinical Pathology, 139(3), $300-308$. doi:10.1309/ajcpegzmjkc42vup.

[13] Canberk, S., Behzatoglu, K., Caliskan, C. K., Gelmez, S., Kayhan, K. C., Aydemir, S. F., ... Schmitt, F. C. (2019). The Role of Telecytology in the Primary Diagnosis of Thyroid Fine-Needle Aspiration Specimens. Acta Cytologica, 64(4), $323-331$. doi:10.1159/000503914. 
[14] Farrell, J. M., Riben, M. W., Staerkel, G. A., Huang, M. L., Dawlett, M., \& Caraway, N. P. (2018). Efficacy of telecytopathology for preliminary assessment of fine-needle aspirations performed at a remote facility. Journal of the American Society of Cytopathology, 7(1), 22-30. doi:10.1016/j.jasc.2017.10.001.

[15] Zanocco, K., Pitelka-Zengou, L., Dalal, S., Elaraj, D., Nayar, R., \& Sturgeon, C. (2013). Routine On-Site Evaluation of Specimen Adequacy during Initial Ultrasound-Guided Fine Needle Aspiration of Thyroid Nodules: A Cost-Effectiveness Analysis. Annals of Surgical Oncology, 20(8), 2462-2467. doi:10.1245/s10434-013-2954-1.

[16] Yeon, J. S., Baek, J. H., Lim, H. K., Ha, E. J., Kim, J. K., Song, D. E., Lee, J. H. (2013). Thyroid Nodules with Initially Nondiagnostic Cytologic Results: The Role of Core-Needle Biopsy. Radiology, 268(1), $274-280$. doi:10.1148/radiol.13122247.

[17] Harel, O. (2007). Inferences on missing information under multiple imputation and two-stage multiple imputation. Statistical Methodology, 4(1), 75-89. doi:10.1016/j.stamet.2006.03.002.

[18] Zhao, L. (2019). SAS for Mixed Models: Introduction and Basic Applications, Walter W.Stroup, George A.Milliken, Elizabeth A.Claassen, Russell D.Wolfinger, Chapman \& Hall/CRC, 2018, 446 pages, e-book, ISBN: 978-1-63526-152-3. International Statistical Review, 87(2), 449-450. doi:10.1111/insr.12348.

[19] Harrell, F. E. (2001). Regression Modeling Strategies. Springer Series in Statistics. doi:10.1007/978-1-4757-3462-1.

[20] Pastorello, R. G., Destefani, C., Pinto, P. H., Credidio, C. H., Reis, R. X., Rodrigues, T. de A., Saieg, M. A. (2018). The impact of rapid on - site evaluation on thyroid fine - needle aspiration biopsy: A 2 - year cancer center institutional experience. Cancer Cytopathology, 126(10), 846-852. doi:10.1002/cncy.22051.

[21] Michael, C. W., Kameyama, K., Kitagawa, W., \& Azar, N. (2020). Rapid on-site evaluation (ROSE) for fine needle aspiration of thyroid: benefits, challenges and innovative solutions. Gland Surgery, 9(5), 1708-1715. doi:10.21037/gs-2019-catp-23.

[22] Lin, D. M., Tracht, J., Rosenblum, F., Kouba, E., Bahl, D., Patel, A., \& Eltoum, I.-E. (2019). Rapid On-Site Evaluation with Telecytology Significantly Reduced Unsatisfactory Rates of Thyroid Fine-Needle Aspiration. American Journal of Clinical Pathology. doi:10.1093/ajcp/aqz164.

[23] Ghofrani, M., Beckman, D., \& Rimm, D. L. (2006). The value of onsite adequacy assessment of thyroid fine-needle aspirations is a function of operator experience. Cancer, 108(2), 110-113. doi:10.1002/cncr.21715. 